\title{
Radiation-Induced Mitotic Delay in L Cells
}

\author{
G. F. WHITMORE, J. E. TILL, AND S. GULYAS \\ Department of Medical Biophysics, University of Toronto, and \\ Ontario Cancer Institute, Toronto, Ontario
}

\begin{abstract}
Whitmore, G. F., Till, J. E., and Gulyas, S. Radiation-Induced Mitotic Delay in L Cells. Radiation Res. 30, pp. 155-171 (1967).

A study has been made of the dependence of the dose-response curve for mitotic delay on the age of the cells at the time of irradiation. The results obtained for randomly dividing cultures with $\mathrm{H}^{3} \mathrm{TdR}$ used to locate the position of the cells in the cell cycle at the time of irradiation indicated that mitotic delay increases from a value of 0.4 to $0.5 \mathrm{~min} / \mathrm{rad}$ for cells irradiated in the $G_{1}$ phase to a value of 2.3 to $2.4 \mathrm{~min} / \mathrm{rad}$ for cells irradiated in $G_{2}$. Cells irradiated in a portion of $S$ gave an intermediate value. The number of cells that attempt the first post-irradiation mitosis decreases with dose more rapidly for cells irradiated in $S$ or $G_{2}$ than for cells irradiated in $G_{1}$. Irradiation of cells that had been partially synchronized by exposure to FUdR yielded results that did not agree with those obtained with randomly dividing cultures.
\end{abstract}

\section{INTRODUCTION}

When mammalian cells in culture are exposed to ionizing radiation, mitotic figures disappear from the population shortly after the irradiation and reappear at a time that is related to the total dose and to a variety of other factors $(1-9)$. It appears well established that, while cells will undergo mitotic delay no matter what their "age" or position in the cell cycle at the time of irradiation, a large part of this delay time will be spent in an extended $G_{2}(2-5,8)$. However, cells irradiated in $G_{1}$ or $S$ may also experience delays in passing through $S$ and into $G_{2}$ $(3,5,8,10,11)$. Except for the work of Terasima and Tolmach (8), using HeLa cells, and that of Dewey and Humphrey (5), using L-P59 cells, little is known about the dependence of the duration of mitotic delay on radiation dose as a function of the position of the cells in the cell cycle at the time of irradiation. Such data can most conveniently be obtained from a synchronized cell population. Thus, in the experiments of Dewey and Humphrey (5), and in those to be reported 
here, tritium thymidine labeling, coupled with autoradiography, was used as a method of labeling $\mathrm{L}$ cells in various parts of the cell cycle so that their behavior at the first mitosis following irradiation could be determined. In addition to the labeling experiments, attempts have been made to use 5 -fluoro- 2 '-deoxyuridine (FUdR) as a means of inducing synchronization (12). The results of both types of experiment indicate that, for $\mathrm{L}$ cells, not only the duration of mitotic delay, but also the number of cells that attempt mitosis following irradiation, is dependent on the position of the cells in the cycle at the time of irradiation. However, the results obtained from measurements of mitotic delay following combined FUdR and radiation treatment are not in complete agreement with results obtained in cultures not exposed to the drug.

\section{MATERIALS AND METHOISS}

\section{$X$-Rays}

The source of $\mathrm{X}$-radiation was a therapy unit (Picker Vanguard) operated at $280 \mathrm{kv}$ and $20 \mathrm{ma}$ and filtered to give a half-value layer of $1.12 \mathrm{~mm} \mathrm{Cu}$ and an average absorbed dose rate of $160 \mathrm{rads} / \mathrm{min}$ in the sample. All irradiations were carried out at $37^{\circ} \mathrm{C}$ on suspension cultures containing 1 to $2 \times 10^{5}$ cells per milliliter, as described previously (11).

\section{Cells and Media}

The L60T cells (12) used were a subline of $\mathrm{L}$ cells grown in suspension culture in CMRL 1066 (13), from which the thymidine and coenzymes had been omitted, supplemented with $10 \%$ horse serum. In certain labeling experiments it was necessary to add TdR concentrations of $10 \mu \mathrm{g} / \mathrm{ml}$ to terminate the uptake of tritiated thymidine $\left(\mathrm{H}^{3} \mathrm{TdR}\right)$. When necessary, FUdR (courtesy of Dr. R. M. Fyfe, Hoffman-La Roche Ltd., Montreal, Quebec) was used at a concentration of $10^{-7} \mathrm{M}$, and Colcemid (courtesy of Dr. C. A. Schaffenburg, Ciba, Co. Ltd., Dorval, Quebec) at a concentration of $10^{-7} \mathrm{gm} / \mathrm{ml}$. To reverse the action of FUdR, the medium was supplemented with $10 \mu \mathrm{g} / \mathrm{ml}$ of thymidine (TdR).

For autoradiographic experiments the medium was supplemented with $\mathrm{H}^{3} \mathrm{TdR}$, usually at a concentration of $0.1 \mu \mathrm{Ci} / \mathrm{ml}$ and with a specific activity of $5.2 \mathrm{CimM}$.

\section{Autoradiography}

The methods used to prepare samples for the determination of mitotic indices as well as the autoradiographic techniques have all been described previously (14). The design of individual experiments will be described in the section on results.

\section{RESULTS}

\section{Measurement of Mitotic Delay}

After the irradiation of mammalian cells, mitotic figures decrease in numbers in the population within 1 to 2 hours, then disappear, and finally reappear at a 
time that is dose-dependent. Measurement of the duration of mitotic delay is facilitated if a mitotic poison such as colchicine or Colcemid is added to the culture sometime between the disappearance and the reappearance of mitotic figures, so that all the cells will be prevented from carrying out successful divisions. Those cells that attempt mitosis in the presence of the inhibitor either will be held at metaphase or will form micronuclei (15). In Fig. 1, the curve marked "Controlmetaphases" shows a typical curve of mitotic index versus time for unirradiated L60T cells in the presence of Colcemid $\left(10^{-7} \mathrm{gm} / \mathrm{ml}\right)$. The curve labeled "Controlmicronuclei" shows that cells containing micronuclei first appear in the population about 6 hours after the addition of Colcemid and that their number steadily increases until by 30 hours almost $90 \%$ of the cells exhibit micronuclei. The curve labeled "Control-total" is the sum of the previous two curves; we have assumed that this curve represents the total fraction of cells in the population that have attempted division, as a function of time. The curve labeled "600 rads-total" is the sum of metaphase figures plus cells containing micronuclei in a population exposed to 600 rads at time zero and given Colcemid $\left(10^{-7} \mathrm{gm} / \mathrm{ml}\right) 1$ hour after irradiation. As will be discussed later, the fact that only $28 \%$ of the cells show either metaphase

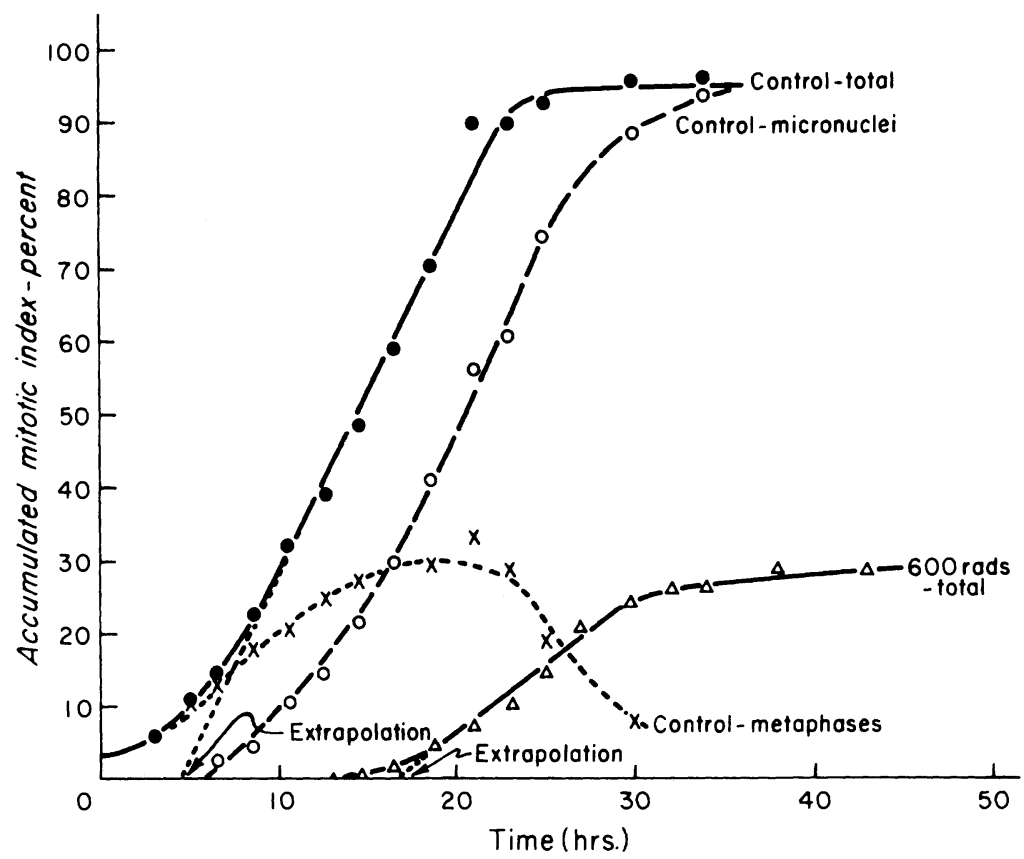

Fig. 1. Accumulation of mitotic figures in the presence of Colcemid $\left(10^{-7} \mathrm{gm} / \mathrm{ml}\right)$, in an unirradiated culture of L60T cells and a culture given 600 rads of X-radiation. The mitotic delay is determined by subtracting the extrapolated value of an unirradiated control population ( 5 hours) from the extrapolated delay of the irradiated population (16 hours). 
figures or micronuclei has been interpreted to mean that after a dose of 600 rads only $28 \%$ of the cells will attempt the first postirradiation division.

In considering curves such as those shown in Fig. 1, the question immediately arises as to what method should be chosen to calculate mitotic delay. One might consider the time at which mitotic figures first reappear in the irradiated cultures, or the time at which the mitotic index reaches some fixed percentage (say 50\%) of the maximum or plateau value, or the time at which the total mitotic index reaches saturation, or the value obtained by extrapolating the steeply rising part of the accumulation curve to zero mitotic index. The results obtained for the dose-response curves for mitotic delay will depend on the choice of criterion. We have chosen to use the value obtained by extrapolation as shown by the dotted line in Fig. 1 because we assumed that it was likely to be indicative of the delay suffered by the first group of cells to reach mitosis after irradiation. Since there is likely to be a certain amount of variation in growth rates of various cells within the population, these results may not be completely representative of the average mitotic delay suffered by all cells, but may instead be an index of the delay in the more rapidly dividing portion of the cell population. To determine the actual mitotic delay, the extrapolated value obtained from an unirradiated control curve was subtracted from the equivalent value obtained for the irradiated population.

If Colcemid is to be used to accumulate mitotic figures, it is first necessary to show that the presence of the drug has no effect on the time of arrival of cells at mitosis in either unirradiated or irradiated cultures. This was shown for both types of culture in an experiment in which cells were labeled with $\mathrm{H}^{3} \mathrm{TdR}(0.1$ $\mu \mathrm{Ci} / \mathrm{ml}$ ) for 6 hours to label cells in $S$ and $G_{2}$. Then labeling was stopped by the addition of unlabeled thymidine. At intervals after the addition of the unlabeled thymidine, paired samples of cells were irradiated with equal doses, Colcemid at $10^{-7} \mathrm{gm} / \mathrm{ml}$ was added to one member of each pair, and the arrival of unlabeled cells at mitosis was observed in both members of each pair. Typical results of such an experiment, with an irradiated culture, are shown in Fig. 2, which indicates the accumulation of unlabeled mitotic figures for an untreated (control) and a Colcemid-treated population, irradiated with 550 rads 15 minutes after the addition of cold thymidine-that is, for cells irradiated in $G_{1}$. Since in both the untreated and the Colcemid-treated populations mitotic figures first appear at approximately 20 hours, and since the peak mitotic frequency in the untreated population occurs at the same time as the half-maximum value in the Colcemid-treated population, there would appear to be little, if any, delaying effect due to Colcemid treatment. Results obtained for cells exposed to Colcemid when irradiated elsewhere in the cell cycle also indicated that the presence of the drug had no important effect on the time required for cells to reach mitosis. These results are in agreement with those obtained for unirradiated cells by Puck and Steffen (16). 


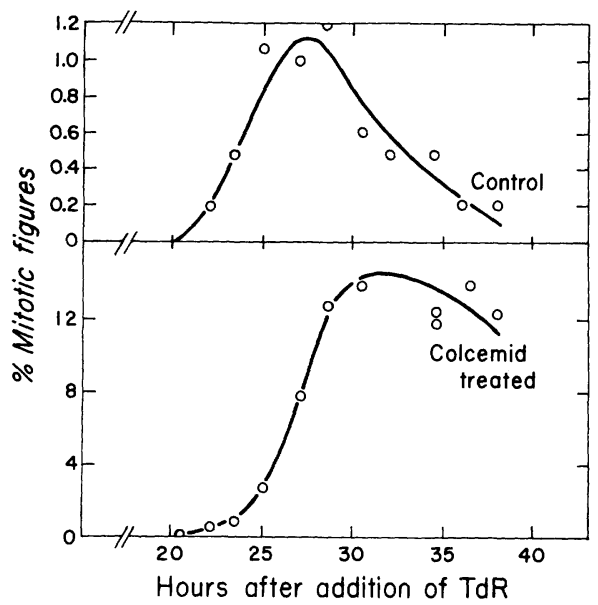

FIG. 2. Accumulation of unlabeled mitotic figures in the presence and in the absence of Colcemid in a culture irradiated with 550 rads. Cells were labeled with $\mathrm{H}^{3} \mathrm{TdR}$ for 6 hours, and then labeling was stopped by dilution of the $\mathrm{H}^{3} \mathrm{TdR}$ with cold TdR. Control culture was irradiated but not treated with Colcemid.

\section{Dose-Response Curves for Mitotic Delay and Division Delay}

If cell counts are carried out on irradiated cultures as a function of time after irradiation, a delay in the resumption of cell division can also be measured. The duration of division delay was estimated by determining the time of onset of cell division, obtained by extrapolating back the rising portion of the growth curve on a semilogarithmic plot, in a manner analogous to that used to estimate mitotic delay (2). Figure 3 shows both mitotic delay and division delay for L-cell cultures irradiated during the logarithmic phase of growth. It can be seen that, for doses above about 100 rads, division delay appears to increase more rapidly with dose than mitotic delay. In both delay curves the rate of increase of delay decreases with increasing dose. For each case, it appears that the initial slope is approximately 2.4 minutes of delay per rad. The curve for mitotic delay appears to approach a final slope of approximately $0.42 \mathrm{~min} / \mathrm{rad}$. The results shown in Fig. 3 are from two separate experiments, and the day-to-day variation can be obtained from comparison of results for equal doses.

The fact that division delay increases more rapidly than mitotic delay may indicate that the duration of mitosis lengthens as a function of dose, as has previously been reported by Harrington (3). There is a possible alternative explanation for the results shown in Fig. 3, which we feel may account for a large part of the difference between the mitotic delay and division delay curves. We will show later in this paper that following a dose of 400 rads only approximately $50 \%$ of the cells in the 


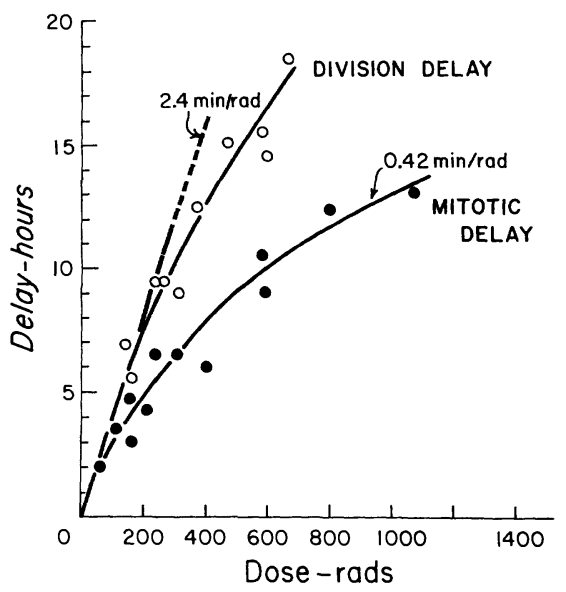

FIG. 3. Mitotic and division delay as a function of dose for an asynchronous population of L60T cells irradiated in the logarithmic growth phase. The value of $2.4 \mathrm{~min} / \mathrm{rad}$ is an estimate of the initial slope of both curves, whereas the value of $0.42 \mathrm{~min} / \mathrm{rad}$ is an estimate of the final slope of the mitotic delay curve.

population will complete one more division. If the nondividing cells remain in the population for some time after irradiation, then we would not expect to see a large increase in the total cell number until the offspring produced by the dividing cells become an appreciable fraction of the total population. This might not occur until sometime after the actual divison process commenced. For a still larger dose of radiation, where the fraction of dividing cells would be smaller, the time taken for the offspring to become an appreciable part of the population would be even longer. If in an irradiated population some of the cells that fail to divide are actually disappearing, then the apparent division delay might be even longer because the new offspring would have to replace the lysed cells before one would see an increase in the population.

\section{Age Dependence of Mitotic Delay}

It is possible to devise a number of different models to explain the form of the mitotic delay or division delay versus dose curve shown in Fig. 3. One explanation is that it results from cells having different sensitivites for mitotic delay because they are at different positions in the cell cycle at the time of irradiation. To investigate this possibility, attempts were made to measure mitotic delays versus dose for cells irradiated in $G_{1}, S$, and $G_{2}$. Initially, autoradiographic techniques were used to observe cells that were in different parts of the cell cycle at the time of irradiation. In the experiments to be reported here, typical values for the durations of the various phases of the L-cell division cycle were $M=0.5$ hour, $G_{1}=4.5$ 
hours, $S=8$ hours, and $G_{2}=3$ hours (12). To observe the mitotic delay for cells irradiated in $S, \mathrm{H}^{3} \mathrm{TdR}(0.1 \mu \mathrm{Ci} / \mathrm{ml})$ was added to a culture of cells that was incubated for 15 minutes. At this time TdR $(10 \mu \mathrm{g} / \mathrm{ml})$ was added to the culture to dilute the $\mathrm{H}^{3} \mathrm{TdR}$ and prevent further detectable labeling, and the population was irradiated. One hour after irradiation Colcemid was added, and, at intervals, samples were removed and autoradiographs were prepared, exposed, developed, and scored for the appearance of labeled mitotic figures. All labeled cells (50\% of the population in this experiment) were assumed to be in $S$ at the time of irradiation. For the observation of cells in $G_{2}, \mathrm{H}^{3} \mathrm{TdR}(0.1 \mu \mathrm{Ci} / \mathrm{ml})$ was added to a culture that was irradiated immediately after addition of the label. Since in this experiment the label was allowed to remain in the culture, the only unlabeled cells that appeared at metaphase would be those cells that were in $G_{2}$ at the time of irradiation. For observations of cells in $G_{1}, \mathrm{H}^{3} \mathrm{TdR}(0.1 \mu \mathrm{Ci} / \mathrm{ml})$ was added to the cultures and allowed to remain for 6 hours. At this time TdR $(10 \mu \mathrm{g} / \mathrm{ml})$ was added to prevent further labeling, and the population was irradiated. Since the combined durations of $G_{2}+M+G_{1}$ were about 8.5 hours, a 6 -hour exposure to $\mathrm{H}^{3} \mathrm{TdR}$ would label all the cells in $S, G_{2}, M$, and the early part of $G_{1}$. Thus, in this experiment, the unlabeled cells reaching metaphase were in late $G_{1}$ at the time of irradiation.

Figure 4 shows the mitotic delay as a function of dose for cells irradiated in $G_{1}$, $S$, and $G_{2}$. For cells irradiated in late $G_{1}$, up to a dose of $1000 \mathrm{R}$, the mitotic delay is a linear function of dose and increases at a rate of $0.47 \mathrm{~min} / \mathrm{rad}$ (see also Table I). It is apparent that $G_{2}$ cells are far more sensitive to radiation-induced mitotic delay

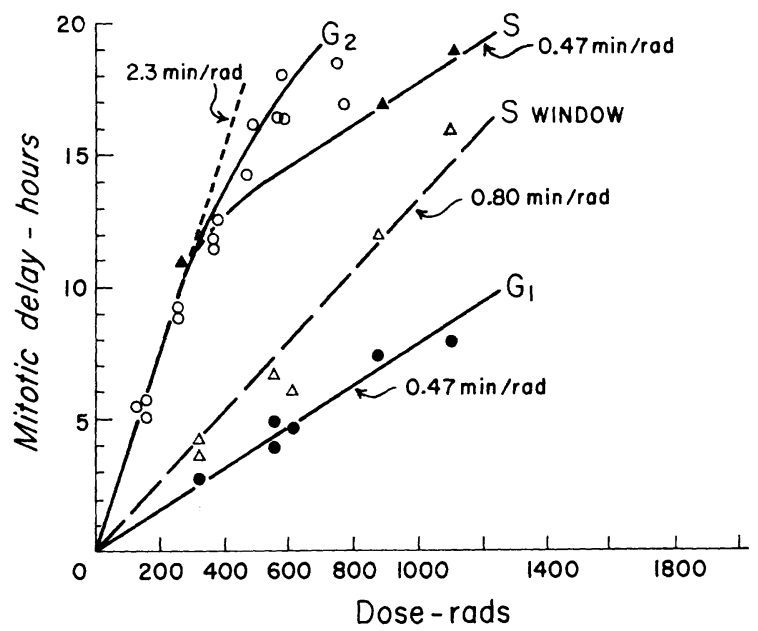

Fig. 4. The mitotic delay as a function of dose for cells irradiated in $G_{1}, S$, and $G_{2}$. The curve marked $S$ window is for a narrow window of cells situated in the early part of the $S$ phase at the time of irradiation (see text). 
than are $G_{1}$ cells. The initial portion of the curve for cells irradiated in $G_{2}$ is a straight line with a slope of $2.3 \mathrm{~min} / \mathrm{rad}$. For doses greater than about $300 \mathrm{rads}$, the slope of the curve decreases. It must be pointed out that the $G_{2}$ population in this experiment does not include those cells that continue through mitosis immediately following irradiation but includes only those that suffer a delay and are held up by Colcemid, administered 1 hour after irradiation.

The results obtained for cell populations irradiated in the $S$ phase are plotted as the curve marked $S$ in $\mathrm{F}: \mathrm{g}$. 4. For a dose of 250 rads the delay appears to be equal to that for cells irradiated in $G_{2}$, while for doses greater than 500 rads the rate of increase in mitotic delay versus dose approaches that of cells irradiated in the $G_{1}$ phase $(0.47 \mathrm{~min} / \mathrm{rad})$. These results may be interpreted to indicate that the cells irradiated in late $S$, which enter mitosis, suffer a mitotic delay similar to that found for cells in $G_{2}$, while those irradiated earlier in $S$ exhibit the same delay as $G_{1}$ cells. It appears that, as the dose of radiation is increased, it is possible for cells that were in early $S$ to overtake cells in late $S$, and so the mitotic delay measured for $S$ cells at very high doses is really a measure of the delay produced in the cells that were in early $S$ phase at the time of irradiation.

Comparison of the data shown in Fig. 4 with the mitotic delay data for randomly dividing cultures shown in Fig. 3 provides strong support for the view that the nonlinear dose-response curve for randomly dividing cultures is due to differences in sensitivity for cells of different ages. The initial slope of the curve for mitotic delay in Fig. 3 is $2.4 \mathrm{~min} / \mathrm{rad}$, which is very similar to the value of $2.3 \mathrm{~min} / \mathrm{rad}$ found for cells irradiated in $G_{2}$ (Fig. 4 and Table I). For larger doses, the slope of the curve in Fig. 3 is $0.42 \mathrm{~min} / \mathrm{rad}$, which is similar to the value of $0.47 \mathrm{~min} / \mathrm{rad}$ found for cells irradiated in $G_{1}$. Again, as in the case of cells irradiated in the $S$ phase, the change in slope seen in the dose-reponse curve for mitotic delay in Fig. 3 appears to be due to cells irradiated at early times in the cell cycle overtaking those irradiated in later phases.

\section{Mitotic Delay for Cells Irradiated in a Portion of $S$}

The techniques described above are useful for observing the responses of the entire population of cells within any one of the three phases-S, $G_{2}$, or $G_{1}$. Since the results obtained for $S$-phase cells indicated a great variation in response among cells in different portions of the $S$ period, an attempt was made to study the mitotic delay suffered by cells irradiated in a limited portion of the $S$ phase. In these experiments, cells in the first half of the $S$ phase were studied by the following method: $\mathrm{H}^{3} \mathrm{TdR}(0.1 \mu \mathrm{Ci} / \mathrm{ml})$ was added to the cultures for a period of 6 hours and then heavily diluted by the addition of unlabeled TdR. At this time, approximately $14 \%$ of the cells were left unlabeled. Since the combined durations of $G_{2}+M+G_{1}$ were about 8.5 hours, these unlabeled cells constituted a narrow "window" of cells that were situated in late $G_{1}$ at the time of addition of cold TdR. At 4.5 hours 
after the addition of cold TdR the cells were irradiated and the mitotic delay of unlabeled cells was determined. Because the duration of $S$ for the culture condition used was approximately 8 hours, this means that the "window" of unlabeled cells was situated somewhere in the first half of the $S$ phase at the time of irradiation. The results obtained are shown as the dashed curve in Fig. 4, marked " $S$ window." The fact that the plot of mitotic delay versus dose is a straight line indicates that, for the part of the $S$ period considered, the variation of mitotic delay versus dose is not a strong function of age. The slope of the curve, $0.80 \mathrm{~min} / \mathrm{rad}$, is intermediate between the values found for cells irradiated in $G_{1}$ and cells irradiated in $G_{2}$. This indicates that the delay suffered by cells irradiated in $S$ may increase continuously as the cells pass through the $S$ period, rather than shifting abruptly from the response characteristic of $G_{1}$ cells to that characteristic of $G_{2}$ cells.

The slopes of the mitotic delay curves given in Figs. 3 and 4 are summarized in Table I.

\section{Relative Numbers of Cells Attempting Mitosis after Irradiation}

In the experiments described above, Colcemid was used to accumulate cells in mitosis. This allows an estimate to be obtained of the percentage of cells that attempted mitosis following irradiation. In unirradiated populations, the accumu-

TABLE I

Mitotic Delay, and Survival of Cells Attempting First Postirradiation Mitosis, for L-Cells Irradiated in Various Portions of the Cell Cycle

\begin{tabular}{lcccc}
\hline Phase of cycle & Method & $\begin{array}{c}\text { Portion of } \\
\text { dose-response curve }\end{array}$ & $\begin{array}{c}\text { Mitotic delay } \\
(\text { min } / \text { rad })\end{array}$ & $\begin{array}{c}D_{37} \text { for mitotic } \\
\text { survival }^{\mathbf{2}}(\text { rads })\end{array}$ \\
\hline All phases $^{\mathrm{b}}$ & - & Initial & 2.4 & - \\
& & Final & 0.42 & 910 \\
\hline$G_{1}$ & $\mathrm{H}^{3} \mathrm{TdR}$ & Initial & 0.47 & 860 \\
$S$ & $\mathrm{H}^{3} \mathrm{TdR}$ & Initial & 2.3 & 140 \\
& & Final & 0.47 & 850 \\
$S$ window & $\mathrm{H}^{3} \mathrm{TdR}$ & Initial & 0.80 & 290 \\
$G_{2}$ & $\mathrm{H}^{3} \mathrm{TdR}$ & Initial & 2.3 & 140 \\
\hline$G_{1}+S$ & FUdR & Initial & 0.45 & 370 \\
$S(1$ hour $)$ & FUdR & Initial & 1.0 & 410 \\
$S(2$ hours $)$ & & Final & 0.45 & 410 \\
& FUdR & Initial & 1.0 & 410 \\
$G_{2}(5$ hours $)$ & FUdR & Final & 0.45 & 920 \\
& & Initial & 1.0 & 920 \\
& & Final & 0.45 & 920 \\
\hline
\end{tabular}

a Estimated from the survival curve for the first postirradiation mitoses (Figs. 5 and 7).

b Randomly dividing, unsynchronized culture. 
lated frequency of mitotic figures and cells with micronuclei in the presence of Colcemid reached a maximum of greater than $80 \%$ in all experiments. The fact that this value does not reach $100 \%$ might be interpreted as indicating that the use of Colcemid does not demonstrate all cells that attempt mitosis. Since in some cases the accumulated frequency of mitotic figures exceeded $95 \%$ (Fig. 1), it was assumed that the Colcemid method at least provides a valid measure of the fraction of rapidly dividing cells in the population that attempts mitosis. In irradiated populations, lower values were observed (see curve marked "600 rads-total" in Fig. 1), and it was found that the percentage of cells that attempted mitosis following irradiation depended on the dose and on the position of the cells in the cell cycle at the time of irradiation. Figure 5 shows the results obtained for the same irradiated cell populations as were used to obtain the data of Figs. 3 and 4 . The results in Fig. 5 are expressed as percentage survival values, relative to the maximum value obtained for an unirradiated population, as a function of radiation dose. Cell populations irradiated in $G_{1}$ (Fig. 5, upper) yielded a survival curve that appeared to be a simple exponential. A survival curve of the same shape was obtained for cells irradiated in a part of $S$, as described in the previous section (" $S$

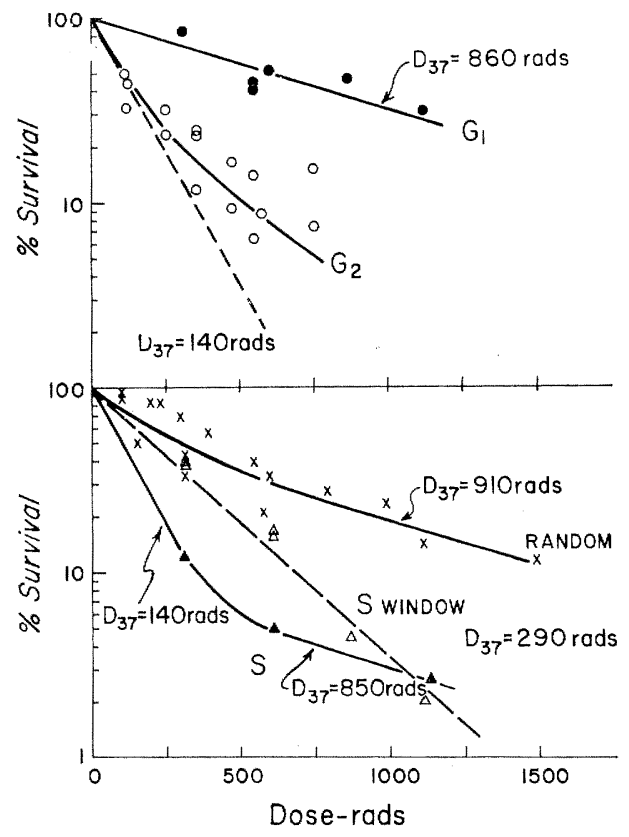

FIG. 5. The percentage of cells attempting the first mitosis after irradiation, as a function of radiation dose. The data were obtained from the same populations as the data in Figs. 3 and 4. 
window," Fig. 5, lower). These were the same populations that showed a linear relationship between mitotic delay and dose (Fig. 4). The remaining populations showed evidence of heterogeneous responses of irradiation, with an indication of a continuous increase in sensitivity as the cells moved from $G_{1}$ through $S$ and into $G_{2}$. Estimates of the $D_{37}$ values (doses required to reduce the percentage survival by a factor of 0.37) for the initial and final portions of the survival curves of Fig. 5 are shown in the figure and are included in Table I. These data provide further support for the view that, for doses in excess of about 300 rads, cells irradiated in early $S$ or $G_{1}$ may reach mitosis before cells irradiated in late $S$ or in $G_{2}$, since the final slopes of the survival curves for randomly dividing populations or for populations irradiated in the $S$ phase approach values similar to the slope of the survival curve for cells irradiated in $G_{1}$.

The finding that the slope of the survival curve for the appearance of first postirradiation mitosis increases as the cells move from $G_{1}$ through $S$ is consistent with the results obtained when $L$ cells irradiated in different portions of the cycle were tested for survival of colony-forming ability (17). Comparisons of the final slopes of the curves obtained with these two different tests of response to radiation indicate that the capacity to enter the first postirradiation mitosis is considerably less sensitive than is the capacity for colony formation. This is the result that would be expected, since it is well known that cells that have lost their capacity for colony formation may be still capable of one or more postirradiation divisions (18).

\section{Mitotic Delay of Cells Synchronized by Treatment with FUdR}

It has been shown $(12,19)$ that FUdR can be used to prevent DNA synthesis in $\mathrm{L}$ cells, and that following reversal of the action of the inhibitor by the addition of TdR a certain degree of synchronization of DNA synthesis and cell division is obtained. An attempt was made to use cells synchronized in this way as another means of studying the age dependence of radiation-induced mitotic delay.

The methods used for synchronization were based on those described previously (12). In brief, FUdR at a concentration of $10^{-7} M$ was added to an exponential culture of $\mathrm{L}$ cells for 16 hours; then the inhibition was reversed, without removal of the FUdR, by the addition of TdR at $10 \mu \mathrm{g} / \mathrm{ml}$. Under these conditions, we have shown that the first $S$ period, which begins immediately after the addition of TdR, is shortened from the normal duration of 8 hours to only about 4 hours (12). This burst of DNA synthesis is followed by a wave of mitoses, which begins 6 hours after the addition of TdR and is essentially complete by 11 hours. Mitotic delay induced in FUdR-treated cells was studied by irradiating the cells at various times, either 1 hour before the addition of TdR, or 1,2 , or 5 hours after addition of TdR. In each case, TdR was added 16 hours after the initiation of FUdR treatment. As before, Colcemid was added after irradiation in order to accumulate mitotic figures, and the extrapolated mitotic delay was estimated in the usual way. 
The results of these experiments are given in Fig. 6. In the case where the cells were irradiated 15 hours after the addition of FUdR-1 hour prior to the addition of TdR-the results indicated by the crosses in Fig. 6 were obtained. At this time, it would be expected that the cells that were not in $S$ at the time of addition of FUdR to the culture (about $50 \%$ of the cells), would have accumulated in late $G_{1}$, immediately prior to $S$. The remaining $50 \%$ of the cells, which were in the $S$ phase at the time of addition of FUdR to the culture, would have been arrested in $S$. The dose-response curve is linear, with little or no evidence of a mixed population response. The slope of the line, $0.45 \mathrm{~min} / \mathrm{rad}$, is approximately equal to the mitotic delay obtained for cells irradiated in $G_{1}$, as determined by the $\mathrm{H}^{3} \mathrm{TdR}$ labeling technique (Fig. 4 and Table I).

As was pointed out above, when TdR is added to FUdR-inhibited cultures, DNA synthesis begins immediately and continues for approximately 4 hours. The results obtained when cells were irradiated at various times after the addition of $\mathrm{TdR}$ are included in Fig. 6. The open and solid circles represent the results of experiments in which the cells were irradiated 1 hour and 5 hours after addition of TdR, respectively. The populations irradiated at 1 hour after addition of TdR should have consisted mainly of cells in $S$, while populations irradiated 5 hours after addition of TdR should have consisted mainly of cells in $G_{2}$, with approximately $10 \%$ of the

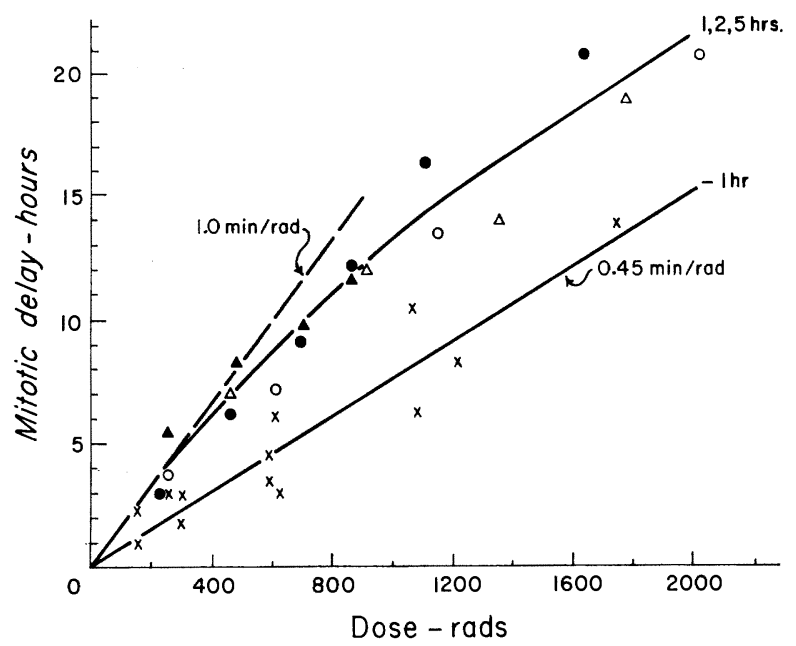

FIG. 6. The mitotic delay as a function of dose for cells treated with $10^{-7} M$ FUdR for 16 hours followed by the addition of $10 \mu \mathrm{g}$ of TdR per milliliter (that is, the mitotic delay for a mixture of late $G_{1}$ and $S$ cells). The times shown are the times of irradiation relative to the time of addition of TdR: $\times$, irradiated 1 hour prior to addition of TdR; $O, \bullet$, irradiated 1 and 5 hours after addition of TdR, respectively. $\triangle, \Delta$, cells held in $G_{1}$ by the action of FUdR (unlabeled cells; see text) and irradiated 2 and 5 hours after addition of TdR, respectively. 
cells still in $S$ (12). However, both populations suffered similar mitotic delays when irradiated with various doses, as may be seen from the data in Fig. 6. Both the open and the closed circles lie along a curve that may be characterized by an initial slope of about $1.0 \mathrm{~min} / \mathrm{rad}$ and a final slope of approximately $0.45 \mathrm{~min} / \mathrm{rad}$ (Table I). This final slope is similar to the slope of the dose-response curve obtained for cells irradiated prior to the addition of TdR. However, the initial slope of 1.0 $\mathrm{min} / \mathrm{rad}$ is considerably less than the initial slope of $2.3 \mathrm{~min} / \mathrm{rad}$ observed when cells that had not been treated with FUdR were irradiated in $S$ or $G_{2}$.

It is possible that the reduced initial slope of the dose-response curve for FUdRtreated cells irradiated in $S$ or $G_{2}$ was due to the fact that the cell population obtained after 16 hours of exposure to FUdR is a heterogeneous one, consisting of cells accumulated prior to $S$ and cells arrested in $S$ (12). This is the case because FUdR inhibits DNA synthesis in cells that were in $S$ at the time of its addition, but does not prevent the progress of cells through the other phases of the cycle. These latter cells accumulate prior to $S$ and should be well synchronized, while the cells that were in $S$ at the time of addition of FUdR remain there and are probably not well synchronized, since they were distributed throughout $S$ at the time of arrest. It was possible that the cells arrested in $S$ might show an anomalous doseresponse curve for mitotic delay, owing to their prolonged inhibition by FUdR, and that if only the cells accumulated prior to $S$ were studied, their dose-response curve would resemble that found for untreated cells. If the cells in $S$ are labeled with $\mathrm{H}^{3} \mathrm{TdR}$ immediately prior to addition of FUdR to the culture, the cells that are not in $S$ at this time, and therefore accumulate prior to $S$ in the presence of FUdR, may be recognized in autoradiographs as unlabeled cells. By scoring only unlabeled mitoses, the dose-response curve for mitotic delay for the well-synchronized portion of the population may be obtained. To accomplish this, an exponentially multiplying population of cells was exposed to $\mathrm{H}^{3} \mathrm{TdR}(0.1 \mu \mathrm{Ci} / \mathrm{ml})$ for 15 minutes, in order to label the cells in $S$. Then the $\mathrm{H}^{3} \mathrm{TdR}$ was removed by centrifuging the cells out of the labeled medium and resuspending them in medium containing FUdR $\left(10^{-7} M\right)$. After 16 hours in the presence of FUdR, cold TdR was added, and aliquots of the population were irradiated 2 hours and 5 hours later. The accumulation of unlabeled mitotic figures in the presence of Colcemid was observed, and mitotic delays were calculated in the usual way. As was pointed out above, since only unlabeled mitotic figures were scored, only cells that were accumulated prior to $S$ by FUdR should have been included in the counts. Two hours after addition of TdR, these cells should have been in the $S$ phase, and after 5 hours they should have been primarily in the $G_{2}$ phase. The results of these experiments are also shown in Fig. 6 ; the data represented by the open and closed triangles were obtained for cells irradiated 2 and 5 hours after addition of TdR, respectively. The data fall along the same dose-response curve as those obtained after FUdR treatment alone. Thus, it seems clear that the mitotic delay suffered 
by cells irradiated in $S$ or $G_{2}$, following accumulation in front of $S$ by exposure to FUdR, is again less than that suffered by cells that have not been exposed to FUdR. It appears that the magnitude of mitotic delay depends not only on the position of cells in the cycle at the time of irradiation but also on other properties of the cells that may be affected by exposure of the cells to FUdR.

\section{Number of Mitoses after FUdR Treatment and Irradiation}

Measurement of the number of cells accumulated in mitosis by Colcemid, following irradiation of FUdR-treated culture, relative to those accumulated in FUdRtreated, unirradiated cultures, yielded the results given in Fig. 7. The FUdR-treated populations studied were the same ones used to obtain the data given in Fig. 6, and the same symbols represent the same populations in the data of both figures. The results given in the upper half of Fig. 7 were for cells irradiated 1 hour prior to addition of TdR ( 15 hours after the addition of FUdR). The results given in the lower half of the figure are for cells exposed for 16 hours to FUdR prior to the addition of TdR, then irradiated 1, 2, or 5 hours after addition of TdR. As in Fig. 6, the open and closed triangles represent the results obtained for cells that had been accumulated prior to $S$ by the action of FUdR (that is, cells not labeled with $\mathrm{H}^{3} \mathrm{TdR}$ ), and the open and closed circles pertain to a mixed population consisting

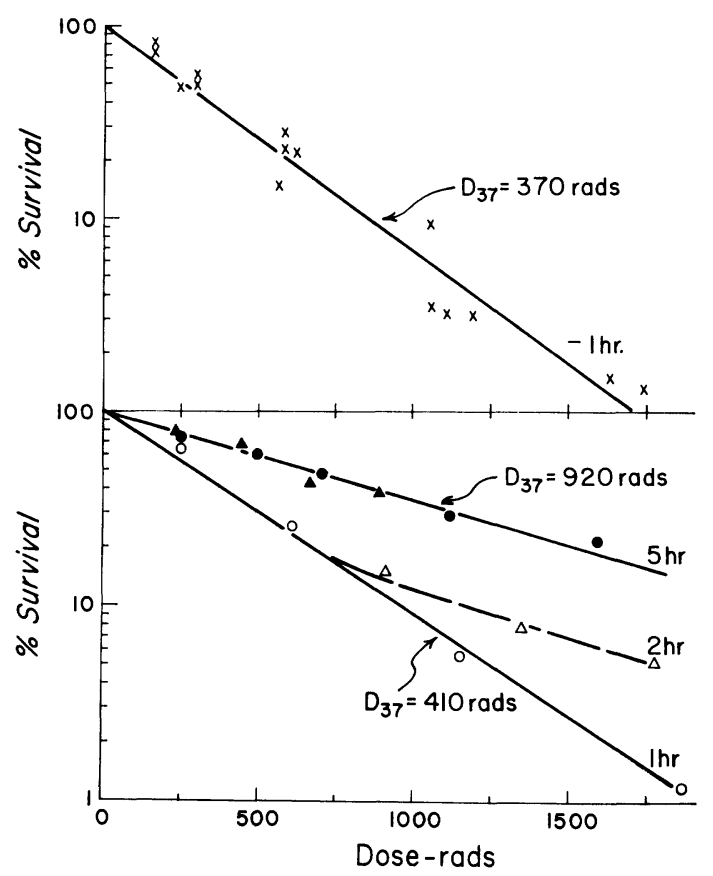

FIG. 7. The percentage of cells reaching the first mitosis after irradiation for the FUdR treated cell populations described in Fig. 6. The symbols used for each population are the same as for Fig. 6. 
of cells that were accumulated prior to $S$ and cells that were arrested in $S$ by FUdR treatment. The $D_{37}$ values obtained from the exponential portions of the survival curves given in Fig. 7 are included in Table I. It was found that for cells irradiated prior to the addition of TdR-cells that were either accumulated prior to $S$ or arrested in $S$-the $D_{37}$ value was about 370 rads. This is much smaller than the $D_{37}$ of 860 rads observed for cells irradiated in $G_{1}$ in the experiments using $\mathrm{H}^{3} \mathrm{TdR}$ labeling (Fig. 5 and Table I), but it is greater than the value of 140 rads found previously for the initial portion of the survival curve for cells irradiated in $S$. One hour after the addition of TdR to the FUdR-treated culture, the survival curve still resembled that obtained prior to the addition of TdR. By 2 hours after addition of TdR, a less-sensitive component could be seen in the survival curve. By 5 hours after addition of TdR, a survival curve with a $D_{37}$ of 920 rads, similar in slope to the less sensitive component of the 2-hour curve, was obtained. Thus, in the FUdR-treated cultures, passage of the cells through $S$ and into $G_{2}$ appeared to result in an increase in the $D_{37}$ of the survival curve, rather than the decrease observed for cells not exposed to FUdR (Fig. 5 and Table I). The $D_{37}$ value obtained for FUdR-treated cells irradiated in $G_{2}(920 \mathrm{rads})$ resembles that obtained for untreated cells irradiated in $G_{1}$ (860 rads). These anomalous results again indicate that the treatment of the cells with FUdR has affected their response to irradiation.

\section{DISCUSSION}

The data presented above, together with the results of others, show that at least five types of response of mammalian cells to irradiation are dependent on the age of the cells at the time of irradiation. These include loss of colony-forming ability $(8,17,20)$, production of chromosomal aberrations (5), inhibition of DNA synthesis $(8,10,11)$, mitotic delay $(5,8$, and Fig. 4$)$, and percentage of cells attempting the first postirradiation mitosis (Fig. 5). The dependence of response on age is not the same in all cases, but the basis for the differences that are observed is not understood.

The dependence of the duration of mitotic delay for $\mathrm{L}$ cells on the age of the cells in the cell cycle at the time of irradiation was studied in the present work by two different methods. In the first type of experiment, randomly dividing cultures were used, and the position of the cells in the cell cycle at the time of irradiation was determined by labeling techniques, using $\mathrm{H}^{3} \mathrm{TdR}$. In the second type of experiment, cells partially synchronized by the action of FUdR were used. The results obtained from the first type of experiment indicate that radiation-induced mitotic delay is greatest for cells irradiated in the $G_{2}$ phase and least for cells irradiated in the $G_{1}$ phase. For cells irradiated in the $S$ phase, delays between the value characteristic of $G_{1}$ cells and that characteristic of $G_{2}$ cells are observed. The results are in good agreement with those of Terasima and Tolmach (8) obtained with HeLa S3 cells, and those of Dewey and Humphrey (5) with L-P59 cells. A complicating factor 
seen in these experiments arose from the fact that mitotic delay increased with the age of the cells in the cell cycle. Because of this, as the dose of radiation was increased, a point was reached after which cells irradiated in $S$ reached mitosis before cells that were in $G_{2}$ at the time of irradiation. After even larger doses, cells irradiated in $G_{1}$ could be the first to divide. This effect resulted in nonlinear doseresponse curves for the mitotic delay suffered by randomly dividing cultures, and by cells irradiated in $S$. It should be stressed that the dose-response curves for mitotic delay are based on an extrapolation procedure that provides an estimate of the minimum delay suffered by cells irradiated in the various phases of the cell cycle. It does not provide information about the distribution of delays suffered by cells of different ages. Such information would be difficult to obtain, since it requires knowledge of the percentage of cells of each age group that reach the first mitosis after irradiation. Some indication of these percentages can be obtained from data on the rates of accumulation of mitotic figures in the presence of Colcemid (see Fig. 1), but these results are also affected by the dependence of mitotic delay on the age of the cells in the cell cycle, which complicates the analysis.

The results of the second type of experiment, in which FUdR was used to obtain partially synchronized cells, are not consistent with the results obtained with randomly dividing cultures. If the only effects of FUdR were to prevent cells in $S$ from continuing DNA synthesis while the remaining cells accumulated at the end of $G_{1}$ immediately prior to $S$, and if the slopes of the dose-response curves for mitotic delay were determined only by the position of the cells relative to the cycle of DNA synthesis and mitosis, then the cells irradiated in $G_{2}$ following exposure to FUdR should have shown a dose-response curve for mitotic delay characterized by an initial slope of about $2.3 \mathrm{~min} / \mathrm{rad}$. Instead, a value of only $1.0 \mathrm{~min} / \mathrm{rad}$ was observed (Table I). Also, when FUdR-treated cultures were irradiated in the $G_{2}$ phase, a much higher proportion of the cells reached the first mitosis after irradiation than was found for cells in $G_{2}$ that had not been exposed to FUdR (Figs. 5 and 7 ). In the former case, this proportion increased as the cells passed from early $S$ into $G_{2}$, while in the latter it did not. The finding from radiation experiments that FUdR treatment affects the properties of cells in the $S$ phase is not surprising, since we have shown (12) that following FUdR treatment the duration of the $S$ phase is reduced from a normal value of about 8 hours to a value of 3 to 4 hours. The results obtained with FUdR-treated cultures are of interest in that they indicate that it may be possible to uncouple radiation-sensitive processes that in normal, randomly dividing populations appear to be closely coupled to the cell cycle. These results are also of value in that they provide another example of the danger of extrapolating results obtained with artificially synchronized cultures to untreated cells.

\section{ACKNOWLEDGMENTS}

We wish to thank the National Cancer Institute of Canada and the U. S. Public Health Service (Grant CA-06229-04) for generous financial support. 


\section{REFERENCES}

1. R. B. Painter and J. S. Robertson, Effect of irradiation and theory of role of mitotic delay on the time course of labeling of HeLa S3 cells with tritiated thymidine. Radiation Res. 11, 206-217 (1959).

2. G. F. Whitmore, C. P. Stanners, J. E. Till, and S. Gulyas, Nucleic acid synthesis and the division cycle in X-irradiated L-strain mouse cells. Biochim. Biophys. Acta 47, 66-77 (1961).

3. H. Harrington, The effect of X-irradiation on the progress of strain U-12 fibroblasts through the mitotic cycle. Ann. N. Y. Acad. Sci. 95, 901-910 (1961).

4. M. YAMADA and T. T. PUCK, Action of radiation on mammalian cells, IV. Reversible mitotic lag in the S3 HeLa cell produced by low doses of X-rays. Proc. Natl. Acad. Sci. U. S. 47, 1181-1191 (1961).

5. W. C. Dewey and R. M. Humphrey, Relative radiosensitivity of different phases of the life cycle of L-P59 mouse fibroblasts and ascites tumor cells. Radiation Res. 16, 503-530, (1962).

6. J. F. Whitfield, R. H. Dixon, and T. Youdale, Prevention of mitotic delay in irradiated suspension cultures of L mouse cells by agmatine. Exptl. Cell Res. 27, 143-147 (1962).

7. D. O. SchNeider and G. F. Whitmore, Comparative effects of neutrons and X-rays on mammalian cells. Radiation Res. 18, 286-306 (1963).

8. T. Terasima and L. J. Tolmach, Variations in several responses of HeLa cells to X-irradiation during the division cycle. Biophys. J. 3, 11-33 (1963).

9. M. M. ElKind, A. HAN, and K. W. Vouz, Radiation response of mammalian cells grown in culture. IV. Dose dependence of division delay and postirradiation growth of surviving and nonsurviving Chinese hamster cells. J. Natl. Cancer Inst. 30, 705-721 (1963).

10. L. G. Lajtha, R. Oliver, T. Kumatori, and F. Ellis, On the mechanism of radiation effect on DNA synthesis. Radiation Res. 8, 1-16 (1958).

11. S. MAK and J. E. TiLL, The effects of X-rays on the progress of L-cells through the cell cycle. Radiation Res. 20, 600-618 (1963).

12. J. E. Till, G. F. Whitmore, and S. Gulyas, Deoxyribonucleic acid synthesis in individual L-strain mouse cells. II. Effects of thymidine starvation. Biochim. Biophys. Acta, 72, 277-289 (1963).

13. R. C. PARKer, Methods of Tissue Culture, 3rd ed., Paul B. Hoeber, New York, 1961.

14. C. P. Stanners and J. E. Till, DNA synthesis in individual L-strain mouse cells. Biochim. Biophys. Acta 37, 406-419 (1960).

15. A. Levan, Colchicine-induced c-mitosis in two mouse ascites tumours. Hereditas 40, 1-64 (1954).

16. T. T. Puck and J. Steffen, Life cycle analysis of mammalian cells. I. A method for localizing metabolic events within the life cycle, and its application to the action of colcemide and sublethal doses of X-irradiation. Biophys. J. 3, 379-397 (1963).

17. G. F. Whitmore, S. Gulyas, and J. Botond, Radiation sensitivity throughout the cell cycle and its relationship to recovery. In Cellular Radiation Biology, Williams \& Wilkins, Baltimore, 1965, 423-441.

18. T. T. Puck and P. I. Marcus, Action of X-rays on mammalian cells. J. Exptl. Med. 103, 653-666 (1956).

19. J. W. Litrlefield, DNA synthesis in partially synchronized L cells. Exptl. Cell Res. 26, 318-326 (1962).

20. W. K. Sinclair and R. A. Morton, X-ray and ultraviolet sensitivity of synchronized Chinese hamster cells at various stages of the cell cycle. Biophys. J. 5, 1-25 (1965). 